\title{
GENERALIZED CENTER AND HYPERCENTER OF A FINITE GROUP
}

\author{
RAM K. AGRAWAL
}

\begin{abstract}
The generalized center of a group $G$ is defined to be the subgroup generated by all elements $g$ of $G$ such that $\langle g\rangle P=P\langle g\rangle$ for all Sylow subgroups $P$ of $G$. This generalizes the concept of the center and quasicenter and leads to the notion of the generalized hypercenter which is defined in the same way as the hypercenter and hyperquasicenter. It is shown that the generalized center is nilpotent and the generalized hypercenter is supersolvable (in fact, the generalized hypercenter is contained in the intersection of the maximal supersolvable subgroups).
\end{abstract}

Generalizing the notion of the center, Ore [10] defined the quasicenter $Q(G)$ of a group $G$ to be the subgroup generated by all elements $g$ of $G$ such that $\langle g\rangle H=H\langle g\rangle$ for all $H \leqslant G$. Mukherjee [9] determined its structure. He also defined and studied the hyperquasicenter $Q^{*}(G)$ of $G$ which generalizes the concept of the hypercenter. Here we define and investigate another center and hypercenter of a finite group.

We say, following Kegel [7], that a subgroup of $G$ is $\pi$-quasinormal in $G$ if it permutes with every Sylow subgroup of $G$ and define the generalized center $Z_{\mathrm{Gn}}(G)$ of $G$ to be the subgroup generated by all elements $g$ of $G$ such that $\langle g\rangle$ is $\pi$-quasinormal in $G$. This leads to the definition of the generalized hypercenter $Z_{\mathrm{Gn}}^{*}(G)$. Let $\left(Z_{\mathrm{Gn}}(G)\right)_{0}=1$ and $\left(Z_{\mathrm{Gn}}(G)\right)_{i+1} /\left(Z_{\mathrm{Gn}}(G)\right)_{i}$ be the generalized center of $G /\left(Z_{\mathrm{Gn}}(G)\right)_{i}$. This yields an ascending chain of characteristic subgroups

$$
\begin{aligned}
1 & =\left(Z_{\mathrm{Gn}}(G)\right)_{0}<\left(Z_{\mathrm{Gn}}(G)\right)_{1}<\left(Z_{\mathrm{Gn}}(G)\right)_{2} \\
& <\cdots<\left(Z_{\mathrm{Gn}}(G)\right)_{m}=Z_{\mathrm{Gn}}^{*}(G) .
\end{aligned}
$$

The terminal member $Z_{G n}^{*}(G)$ is called the generalized hypercenter.

The main results are: (1) If $\langle g\rangle$ is $\pi$-quasinormal in $G$, so is every subgroup of $\langle g\rangle$; (2) $Z_{\mathrm{Gn}}(G)$ is nilpotent; (3) If $G=H \times K$, then

$$
Z_{\mathrm{Gn}}(G)=Z_{\mathrm{Gn}}(H) \times Z_{\mathrm{Gn}}(K) \quad \text { and } Z_{\mathrm{Gn}}^{*}(G)=Z_{\mathrm{Gn}}^{*}(H) \times Z_{\mathrm{Gn}}^{*}(K) ;
$$

(4) $Z_{\mathrm{Gn}}^{*}(G)$ is supersolvable; (5) $Z_{\mathrm{Gn}}^{*}(G)$ is contained in the intersection of the maximal supersolvable subgroups of $G$; and $(6) Z_{\mathrm{Gn}}^{*}(G)$ is the product of all generalized hypercentral subgroups of $G$.

Received by the editors May 14, 1975.

AMS (MOS) subject classifications (1970). Primary 20D40.

Key words and phrases. $\pi$-quasinormal subgroup, generalized center, generalized central element, generalized hypercenter, generalized hypercentral subgroup. 
Throughout, only finite groups are considered.

\section{Generalized center.}

Definitions. Subgroups $H$ and $K$ of $G$ permute if $H K=K H$. A subgroup of $G$ is called $\pi$-quasinormal (quasinormal) in $G$ if it permutes with all Sylow subgroups (all subgroups) of $G$. The quasicenter $Q(G)$ of $G$ is the subgroup generated by all elements $g$ of $G$ such that $\langle g\rangle$ is quasinormal in $G$.

The following results on $\pi$-quasinormality are frequently used later.

(1.1) Kegel [7]. If $H \leqslant K \leqslant G$ and $H$ is $\pi$-quasinormal in $G$, then $H$ is $\pi$ quasinormal in $K$.

(1.2) Kegel [7]. A $\pi$-quasinormal subgroup of $G$ is subnormal in $G$.

(1.3) Kegel [7]. If $\theta$ is a homomorphism from $G$ onto $G^{\theta}$ and $H$ is a $\pi$ quasinormal subgroup of $G$, then $H^{\theta}$ is a $\pi$-quasinormal subgroup of $G^{\theta}$.

(1.4) Let $p$ be a prime dividing $|G|$ and $H$ be a $p$-subgroup of $G$. If $H$ is $\pi$ quasinormal in $G$, then $H \triangleleft H G_{q}$ for every Sylow $q$-subgroup $G_{q}$ of $G$ with $q \neq p$.

Proof. Since $H G_{q}=G_{q} H, H G_{q}$ is a subgroup. By (1.1), $H$ is $\pi$-quasinormal in $H G_{q}$. Hence by (1.2), $H$ is a subnormal Sylow $p$-subgroup of $H G_{q}$. This implies that $H$ is normal in $H G_{q}$.

Definition. The generalized center $Z_{\mathrm{Gn}}(G)$ of a group $G$ is the subgroup generated by all elements $g$ of $G$ such that $\langle g\rangle$ is $\pi$-quasinormal in $G$. Such elements shall be called generalized central elements of $G$.

REMARK. $Z_{\mathrm{Gn}}(G)$ is a characteristic subgroup of $G$ which contains the quasicenter $Q(G)$ and the center $Z(G)$ of $G$. The following example shows that $Z_{\mathrm{Gn}}(G)$ can be nontrivial even though $Q(G)$ is trivial.

EXAMPLE 1. Let $G$ be the symmetric group $S_{3}$ wreathed by the cyclic group $C_{3}$ of order 3. That is, $G$ is the direct product of three copies of $S_{3}$ extended by an automorphism of order 3 which permutes the copies in a cycle. For this group, $Q(G)=1$ but $Z_{\mathrm{Gn}}(G)$ has order 27 .

Subgroups of a normal cyclic subgroup are normal in the group. A similar result holds for the subgroups of a $\pi$-quasinormal cyclic subgroup.

THEOREM 1.5. If $\langle g\rangle$ is $\pi$-quasinormal in $G$, then every subgroup of $\langle g\rangle$ is also $\pi$-quasinormal in $G$.

Proof. Let $\left\langle g^{n}\right\rangle$ be a subgroup of $\langle g\rangle$, where $n$ is an integer. To prove the theorem, we must show that $\left\langle g^{n}\right\rangle G_{p}=G_{p}\left\langle g^{n}\right\rangle$ for an arbitrary but fixed Sylow $p$-subgroup $G_{p}$ of $G$. By (1.2), $\langle g\rangle$ is subnormal in $G$. Hence $\left\langle g^{n}\right\rangle$ is subnormal in $G$. Let $P$ be the Sylow $p$-subgroup of $\left\langle g^{n}\right\rangle$ and $K$ be the $p$ complement of $\left\langle g^{n}\right\rangle$. Now, since $P$ is a subnormal $p$-subgroup of $G$, it follows that $P \leqslant G_{p}$ and therefore $P G_{p}=G_{p} P=G_{p}$. Let $H$ be the $p$-complement of $\langle g\rangle$. Then $H$ is subnormal in $G$ and hence $H$ is subnormal in $\langle g\rangle G_{p}$. This means that $H$ is a subnormal Hall subgroup of $\langle g\rangle G_{p}$, which implies that $H \triangleleft\langle g\rangle G_{p}$. But $K \leqslant H$ and $H$ is cyclic. Hence $K \triangleleft\langle g\rangle G_{p}$ and so $K G_{p}$ $=G_{p} K$. Since $\left\langle g^{n}\right\rangle=P \times K$, it follows that $\left\langle g^{n}\right\rangle$ and $G_{p}$ permute.

REMARK. Using (1.3), one can verify that if $G \cong \bar{G}$ under the isomorphism $\theta$, then $\left(Z_{\mathrm{Gn}}(G)\right)^{\theta}=Z_{\mathrm{Gn}}(\bar{G})$.

The following example shows that every subgroup of $Z_{\mathrm{Gn}}(G)$ need not be 
$\pi$-quasinormal in $G$ and that if $\langle a\rangle$ and $\langle b\rangle$ are $\pi$-quasinormal in $G$, then $\langle a b\rangle$ is not always $\pi$-quasinormal in $G$.

EXAMPLE 2. Let $G=\langle a, b\rangle\langle x\rangle$ with $a^{3}=b^{3}=1, a b=b a, a^{x}=a^{2}, b^{x}$ $=b$ and $x^{2}=1$. Clearly, $Z_{\mathrm{Gn}}(G)=\langle a, b\rangle$. But $\langle a b\rangle$ is not $\pi$-quasinormal in $G$ since $\langle a b\rangle\langle x\rangle \neq\langle x\rangle\langle a b\rangle$.

Proposition 1.6. If $G=H \times K$, then $Z_{\mathrm{Gn}}(G)=Z_{\mathrm{Gn}}(H) \times Z_{\mathrm{Gn}}(K)$.

Proof. First we show that $Z_{\mathrm{Gn}}(H) \times Z_{\mathrm{Gn}}(K) \leqslant Z_{\mathrm{Gn}}(G)$. Let $h$ be an element of $H$ such that $\langle h\rangle$ is $\pi$-quasinormal in $H$ and $G_{p}$ be a Sylow $p$ subgroup of $G$. Since $G_{p}=H_{p} \times K_{p}$ for some $H_{p}$ and $K_{p}$ and $\langle h\rangle$ is centralized by $K$, it follows that $\langle h\rangle G_{p}=G_{p}\langle h\rangle$. Hence, $\langle h\rangle$ is $\pi$-quasinormal in $G$ and so $h \in Z_{\mathrm{Gn}}(G)$. Thus $Z_{\mathrm{Gn}}(H) \leqslant Z_{\mathrm{Gn}}(G)$. Similarly, $Z_{\mathrm{Gn}}(K) \leqslant Z_{\mathrm{Gn}}(G)$.

Next to show that $Z_{\mathrm{Gn}}(G) \leqslant Z_{\mathrm{Gn}}(H) \times Z_{\mathrm{Gn}}(K)$, let $g$ be an element of $G$ such that $\langle g\rangle$ is $\pi$-quasinormal in $G$. Let $P$ be the Sylow $p$-subgroup of $\langle g\rangle$. Clearly, $P=\langle h k\rangle$ for some $p$-elements $h \in H$ and $k \in K$. We will show that $\langle h\rangle$ is $\pi$-quasinormal in $H$. For this, let $H_{q}$ be any Sylow $q$-subgroup of $H$, $q \neq p$ and $x$ be an element of $H_{q}$. By Theorem 1.5, $P$ is $\pi$-quasinormal in $G$. Hence it follows from (1.4) that $P \triangleleft P G_{q}$ for every Sylow $q$-subgroup $G_{q}$ of $G$, $q \neq p$. In particular, $x^{-1}(h k) x=(h k)^{n}=h^{n} k^{n}$ for some integer $n$. This implies that $x^{-1} h x=h^{n}$ and so $\langle h\rangle$ permutes with every $H_{q}$ for primes $q \neq p$. Since $P$ is $\pi$-quasinormal in $G, P=\langle h k\rangle \leqslant \cap G_{p}=\cap\left(H_{p} \times K_{p}\right)$. Hence $h$ belongs to every Sylow $p$-subgroup $H_{p}$ of $H$. Consequently, $\langle h\rangle H_{p}=H_{p}\langle h\rangle$ $=H_{p}$ which implies that $\langle h\rangle$ is $\pi$-quasinormal in $H$ and so $h \in Z_{\mathrm{Gn}}(H)$. Similarly $k \in Z_{\mathrm{Gn}}(K)$. Thus $P \leqslant Z_{\mathrm{Gn}}(H) \times Z_{\mathrm{Gn}}(K)$. Since $\langle g\rangle$ is the direct product of its Sylow subgroups, it follows that $\langle g\rangle \leqslant Z_{\mathrm{Gn}}(H) \times Z_{\mathrm{Gn}}(K)$. This completes the proof.

REMARK. Note that if $G$ is nilpotent, then $Z_{\mathrm{Gn}}(G)=G$ but the quasicenter $Q(G)$ is not necessarily $G$.

To determine the structure of $Z_{\mathrm{Gn}}(G)$, we need the following:

LemMA 1.7. Every Sylow subgroup of $Z_{\mathrm{Gn}}(G)$ of a group $G$ is generated by generalized central elements of $G$.

Proof. Let $\left|Z_{\mathrm{Gn}}(G)\right|=p_{1}^{\alpha_{1}} p_{2}^{\alpha_{2}} \cdots p_{n}^{\alpha_{n}}$, where $p_{1}, p_{2}, \ldots, p_{n}$ are primes. For $1 \leqslant i \leqslant n$, denote by $S_{p_{i}}$ the subgroup generated by all $p_{i}$-elements of $G$ that are generalized central elements of $G$. Clearly, each $S_{p_{i}}$ is $\pi$-quasinormal in $G$ and hence is subnormal in $G$. Furthermore, since a generalized central $p_{i}{ }^{-}$ element of $G$ belongs to every Sylow $p_{i}$-subgroup $G_{p_{i}}$ of $G$, it follows that $S_{p_{i}} \leqslant \cap G_{p_{i}}$. Therefore, $S_{p_{i}}$ is a $p_{i}$-subgroup of $G$.

Let $p_{j}$ and $p_{k}$ be any two different primes dividing the order of $Z_{\mathrm{Gn}}(G)$. Then, by the preceding paragraph and (1.4), $S_{p_{j}} \triangleleft S_{p_{j}} G_{p_{k}}$. Hence $S_{p_{j}}$ and $S_{p_{k}}$ permute. Thus, $S_{p_{j}} S_{p_{k}}$ is a subgroup for all primes $p_{j}$ and $p_{k}$ with $p_{j} \neq p_{k}$ and $1 \leqslant j, k \leqslant n$. Therefore, $S_{p_{1}} S_{p_{2}} \cdots S_{p_{n}}$ is a subgroup which is contained in $Z_{\mathrm{Gn}}(G)$. Now, let $g$ be any generalized central element of $G$. Since $\langle g\rangle$ is the direct product of its Sylow subgroups each of which is cyclic and $\pi$ quasinormal in $G$, it follows that every Sylow subgroup of $\langle g\rangle$ is contained in some $S_{p_{i}}$. Hence $\langle g\rangle \leqslant S_{p_{1}} S_{p_{2}} \cdots S_{p_{n}}$, which implies that $Z_{\mathrm{Gn}}(G)$ 
$\leqslant S_{p_{1}} S_{p_{2}} \cdots S_{p_{n}}$. Thus $Z_{\mathrm{Gn}}(G)=S_{p_{1}} S_{p_{2}} \cdots S_{p_{n}}$. From this, the assertion in the lemma follows immediately.

THEOREM 1.8. $Z_{\mathrm{Gn}}(G)$ of a group $G$ is nilpotent.

Proof. Let $P$ be a Sylow subgroup of $Z_{\mathrm{Gn}}(G)$. By Lemma 1.7, $P$ is subnormal (in fact $\pi$-quasinormal) in $G$. Hence $P$ is subnormal in $Z_{\mathrm{Gn}}(G)$ and so $P \triangleleft Z_{\mathrm{Gn}}(G)$. Thus $Z_{\mathrm{Gn}}(G)$ is nilpotent.

REMARK. Clearly, every Sylow subgroup of $Z_{\mathrm{Gn}}(G)$ is characteristic in $G$ and $G$ is nilpotent if and only if $G=Z_{\mathrm{Gn}}(G)$.

\section{Generalized hypercenter.}

Definition. For a group $G$, let $\left(Z_{\mathrm{Gn}}(G)\right)_{0}=1$ and $\left(Z_{\mathrm{Gn}}(G)\right)_{i+1} /\left(Z_{\mathrm{Gn}}(G)\right)_{i}$ be the generalized center of $G /\left(Z_{\mathrm{Gn}}(G)\right)_{i}$. The generalized hypercenter $Z_{\mathrm{Gn}}^{*}(G)$ of $G$ is the terminal member of the chain of characteristic subgroups

$$
\begin{aligned}
1 & =\left(Z_{\mathrm{Gn}}(G)\right)_{0}<Z_{\mathrm{Gn}}(G)=\left(Z_{\mathrm{Gn}}(G)\right)_{1}<\left(Z_{\mathrm{Gn}}(G)\right)_{2} \\
& <\cdots<\left(Z_{\mathrm{Gn}}(G)\right)_{m}=Z_{\mathrm{Gn}}^{*}(G) .
\end{aligned}
$$

REMARK. $Z_{\mathrm{Gn}}^{*}(G)$ contains the hypercenter and hyperquasicenter of $G$. Example 1 of $\$ 1$ shows that the generalized hypercenter of a group can be nontrivial even though its hyperquasicenter is trivial. Also note that $Z_{\mathrm{Gn}}^{*}(G)$ is not necessarily nilpotent as shown by the symmetric group on 3 letters. However, we shall show that $Z_{\mathrm{Gn}}^{*}(G)$ is supersolvable.

THEOREM 2.1. For a group $G, Z_{\mathrm{Gn}}^{*}(G)=\bigcap\left\{N \mid N \triangleleft G\right.$ and $Z_{\mathrm{Gn}}(G / N)$ $=\overline{1}$ \}.

Proof. Consider the chain

$$
1=\left(Z_{\mathrm{Gn}}(G)\right)_{0}<\left(Z_{\mathrm{Gn}}(G)\right)_{1}<\cdots<\left(Z_{\mathrm{Gn}}(G)\right)_{m}=Z_{\mathrm{Gn}}^{*}(G)
$$

and let $T=\cap\left\{N \mid N \triangleleft G\right.$ and $\left.Z_{\mathrm{Gn}}(G / N)=\overline{1}\right\}$. Then $T \leqslant Z_{\mathrm{Gn}}^{*}(G)$ since $Z_{\mathrm{Gn}}\left(G / Z_{\mathrm{Gn}}^{*}(G)\right)=\overline{1}$. To prove $Z_{\mathrm{Gn}}^{*}(G) \leqslant T$, let $K$ be any element of $\left\{N \mid N \triangleleft G\right.$ and $\left.Z_{\mathrm{Gn}}(G / N)=\overline{1}\right\}$. It is easy to see that $\left(Z_{\mathrm{Gn}}(G)\right)_{1}=Z_{\mathrm{Gn}}(G)$ $\leqslant K$. Next we assume that $\left(Z_{\mathrm{Gn}}(G)\right)_{i} \leqslant K, 1 \leqslant i<m$, and show that $\left(Z_{\mathrm{Gn}}(G)\right)_{i+1} \leqslant K$. Let $g\left(Z_{\mathrm{Gn}}(G)\right)_{i}$ be any generalized central element of $G /\left(Z_{\mathrm{Gn}}(G)\right)_{i}$. Since $G / K$ is a homomorphic image of $G /\left(Z_{\mathrm{Gn}}(G)\right)_{i}$, it follows that $g K$ is a generalized central element of $G / K$. But $Z_{\mathrm{Gn}}(G / K)=\overline{1}$. Hence $g \in K$ which implies that

$$
Z_{\mathrm{Gn}}\left(G /\left(Z_{\mathrm{Gn}}(G)\right)_{i}\right)=\left(Z_{\mathrm{Gn}}(G)\right)_{i+1} /\left(Z_{\mathrm{Gn}}(G)\right)_{i} \leqslant K /\left(Z_{\mathrm{Gn}}(G)\right)_{i} .
$$

Thus $\left(Z_{\mathrm{Gn}}(G)\right)_{i+1} \leqslant K$ and so $Z_{\mathrm{Gn}}^{*}(G) \leqslant K$. The rest is obvious.

The next proposition follows from the preceding theorem.

Proposition 2.2. Let $N$ be a normal subgroup of $G$. Then $Z_{\mathrm{Gn}}^{*}(G) N / N$ $\leqslant Z_{\mathrm{Gn}}^{*}(G / N)$.

The inclusion here can be proper as shown by the alternating group of degree 4 and its Sylow 2-subgroup. 
Proposition 2.3. Let $T$ be a normal subgroup of $G$ such that $T \leqslant Z_{\mathrm{Gn}}^{*}(G)$. Then $Z_{\mathrm{Gn}}^{*}(G / T)=Z_{\mathrm{Gn}}^{*}(G) / T$.

Proof. Consider the chain

$$
\overline{1}=T / T<W_{1} / T<W_{2} / T<\cdots<W_{m} / T=Z_{\mathrm{Gn}}^{*}(G / T),
$$

where

$$
W_{1} / T=Z_{\mathrm{Gn}}(G / T) \text { and } W_{i} / T / W_{i-1} / T=Z_{\mathrm{Gn}}\left(G / T / W_{i-1} / T\right)
$$

for $i=2,3, \ldots, m$. Since $T \leqslant Z_{\mathrm{Gn}}^{*}(G)$, Proposition 2.2 implies that $Z_{\mathrm{Gn}}^{*}(G)$ $\leqslant W_{m}$. To prove $W_{m} \leqslant Z_{\mathrm{Gn}}^{*}(G)$, we first show that $W_{1} \leqslant Z_{\mathrm{Gn}}^{*}(G)$. For this, let $g T$ be any generalized central element of $G / T$. Then $g Z_{\mathrm{Gn}}^{*}(G)$ is a generalized central element of $G / Z_{\mathrm{Gn}}^{*}(G)$ since $T \leqslant Z_{\mathrm{Gn}}^{*}(G)$. Hence $g$ $\in Z_{\mathrm{Gn}}^{*}(G)$ which implies that $W_{1} \leqslant Z_{\mathrm{Gn}}^{*}(G)$. We now assume that $W_{i}$ $\leqslant Z_{\mathrm{Gn}}^{*}(G), 2 \leqslant i<m$, and will show that $W_{i+1} \leqslant Z_{\mathrm{Gn}}^{*}(G)$.

Since $G / T / W_{i} / T \cong G / W_{i}$, it follows easily that $Z_{\mathrm{Gn}}\left(G / W_{i}\right)=W_{i+1} / W_{i}$. Now replacing $T$ by $W_{i}$ in the argument used above, one can show that $W_{i+1} \leqslant Z_{\mathrm{Gn}}^{*}(G)$. Hence $W_{m} \leqslant Z_{\mathrm{Gn}}^{*}(G)$.

Let $H$ be a subgroup of $G$. In general, there is no relationship between $Z_{\mathrm{Gn}}^{*}(G)$ and $Z_{\mathrm{Gn}}^{*}(H)$. But if $Z_{\mathrm{Gn}}^{*}(G) \leqslant H$, then we have

Proposition 2.4. If $H$ is a subgroup of $G$ and $Z_{\mathrm{Gn}}^{*}(G) \leqslant H$, then $Z_{\mathrm{Gn}}^{*}(G)$ $\leqslant Z_{\mathrm{Gn}}^{*}(H)$. In particular, $Z_{\mathrm{Gn}}^{*}\left(Z_{\mathrm{Gn}}^{*}(G)\right)=Z_{\mathrm{Gn}}^{*}(G)$.

One can show that if $G$ and $\bar{G}$ are isomorphic under the map $\alpha$, then $Z_{\mathrm{Gn}}^{*}(\bar{G})$ is the image of $Z_{\mathrm{Gn}}^{*}(G)$ under $\alpha$. Using this and Proposition 1.6, we prove the following.

Proposition 2.5. If $G=H \times K$, then $Z_{\mathrm{Gn}}^{*}(G)=Z_{\mathrm{Gn}}^{*}(H) \times Z_{\mathrm{Gn}}^{*}(K)$.

PRoof. We use induction on $|G|$. Clearly, we may assume that $Z_{\mathrm{Gn}}(G)$ $=Z_{\mathrm{Gn}}(H) \times Z_{\mathrm{Gn}}(K) \neq 1$. By induction and Proposition 2.3,

$$
\begin{aligned}
Z_{\mathrm{Gn}}^{*}\left(H / Z_{\mathrm{Gn}}(H) \times K / Z_{\mathrm{Gn}}(K)\right) & =Z_{\mathrm{Gn}}^{*}\left(H / Z_{\mathrm{Gn}}(H)\right) \times Z_{\mathrm{Gn}}^{*}\left(K / Z_{\mathrm{Gn}}(K)\right) \\
& =Z_{\mathrm{Gn}}^{*}(H) / Z_{\mathrm{Gn}}(H) \times Z_{\mathrm{Gn}}^{*}(K) / Z_{\mathrm{Gn}}(K) .
\end{aligned}
$$

Let $G / Z_{\mathrm{Gn}}(G)$ and $H / Z_{\mathrm{Gn}}(H) \times K / Z_{\mathrm{Gn}}(K)$ be isomorphic under the map $\theta$. Then,

$$
\begin{aligned}
& Z_{\mathrm{Gn}}^{*}(H) / Z_{\mathrm{Gn}}(H) \times Z_{\mathrm{Gn}}^{*}(K) / Z_{\mathrm{Gn}}(K) \\
&=\left(Z_{\mathrm{Gn}}^{*}\left(G / Z_{\mathrm{Gn}}(G)\right)\right)^{\theta}=\left(Z_{\mathrm{Gn}}^{*}(G) / Z_{\mathrm{Gn}}(G)\right)^{\theta} .
\end{aligned}
$$

Now apply $\theta^{-1}$ to get the desired result.

The next result is needed to determine the structure of $Z_{\mathrm{Gn}}^{*}(G)$.

THeORem 2.6. $Z_{\mathrm{Gn}}^{*}(G)$ of a group $G$ has the Sylow tower property of supersolvable groups.

Proof. We use induction on $|G|$. Thus, in view of Proposition 2.4, we may 
assume that $Z_{\mathrm{Gn}}^{*}(G)=G$. Let $p$ be the largest prime divisor of $|G|$ and $G_{p}$ be a Sylow $p$-subgroup of $G$. We shall show that $G_{p} \triangleleft G$.

If $p$ does not divide $\left|G / Z_{\mathrm{Gn}}(G)\right|$, then $G_{p} \leqslant Z_{\mathrm{Gn}}(G)$. But $Z_{\mathrm{Gn}}(G)$ is nilpotent and so $G_{p} \triangleleft G$. On the other hand, if $p$ divides $\left|G / Z_{\mathrm{Gn}}(G)\right|$, then by induction $\left(G / Z_{\mathrm{Gn}}(G)\right)_{p}$ is normal in $G / Z_{\mathrm{Gn}}(G)$ since $Z_{\mathrm{Gn}}^{*}\left(G / Z_{\mathrm{Gn}}(G)\right)$ $=G / Z_{\mathrm{Gn}}(G)$. Hence $G_{p} Z_{\mathrm{Gn}}(G) \triangleleft G$. But the Sylow subgroups of $Z_{\mathrm{Gn}}(G)$ are normal in $G$ and so $G_{p} Z_{\mathrm{Gn}}(G)=G_{p} L \triangleleft G$, where $L$ is the $p$-complement of $Z_{\mathrm{Gn}}(G)$. To prove that $G_{p} \triangleleft G$, it suffices to show that $L \leqslant N_{G}\left(G_{p}\right)$ since $G=G_{p} L N_{G}\left(G_{p}\right)=L N_{G}\left(G_{p}\right)$ by Frattini's Lemma. For this, let $x$ be any $q$ element of $G$ for a prime $q \neq p$ such that $\langle x\rangle$ is $\pi$-quasinormal in $G$. By (1.4), $\langle x\rangle \triangleleft\langle x\rangle G_{p}$. Let $g$ be any element of $G_{p}$. Then $\langle x\rangle\langle g\rangle$ is a supersolvable subgroup of $G$ and $\langle x\rangle \triangleleft\langle x\rangle\langle g\rangle$. Since $p\rangle q,\langle g\rangle$ is also normal in $\langle x\rangle\langle g\rangle$. Hence $g$ and $x$ centralize each other and so $x \in N_{G}\left(G_{p}\right)$. It follows from Lemma 1.7 that $L \leqslant N_{G}\left(G_{p}\right)$. Thus $G_{p} \triangleleft G$.

Now consider $G / G_{p}$. By induction, $Z_{\mathrm{Gn}}^{*}\left(G / G_{p}\right)=G / G_{p}$ has the Sylow tower property of supersolvable groups, which means that $G=Z_{\mathrm{Gn}}^{*}(G)$ has this property. This proves the theorem.

We are now ready to prove

THEOREM 2.7. $Z_{\mathrm{Gn}}^{*}(G)$ of a group $G$ is supersolvable.

Proof. We use induction on $|G|$. Since $Z_{\mathrm{Gn}}^{*}\left(Z_{\mathrm{Gn}}^{*}(G)\right)=Z_{\mathrm{Gn}}^{*}(G)$, we may assume that $Z_{\mathrm{Gn}}^{*}(G)=G$. This means that every factor group $G / K$ for $K \neq 1$ is supersolvable by induction because $Z_{\mathrm{Gn}}^{*}(G / K)=Z_{\mathrm{Gn}}^{*}(G) / K=G / K$.

If the Frattini subgroup $\Phi(G)$ of $G$ is not identity, then $G / \Phi(G)$ is supersolvable and, therefore, $G$ is supersolvable. So we may assume that $\Phi(G)=1$. Let $p$ be the largest prime divisor of $|G|$. By Theorem 2.6, the Sylow $p$-subgroup $P$ of $G$ is normal in $G$. Hence $\Phi(P) \leqslant \Phi(G)$ and so $\Phi(P)=1$. This means that $P$ is elementary abelian. Let $M$ be a maximal subgroup of $G$. We will show that $[G: M]$ is a prime.

By Theorem 2.6, $G$ is solvable. Hence $[G: M]$ is a power of a prime. If $[G: M]$ is not a power of $p$, then $P \leqslant M$ and, since $G / P$ is supersolvable, $[G / P: M / P]=[G: M]$ is a prime. On the other hand, if $[G: M]$ is a power of $p$, we consider $Z_{\mathrm{Gn}}(G)$ and proceed as follows: Clearly, $Z_{\mathrm{Gn}}(G) \neq 1$. If a prime $q \neq p$ divides $\left|Z_{\mathrm{Gn}}(G)\right|$, then the Sylow $q$-subgroup $\bar{Q}$ of $Z_{\mathrm{Gn}}(G)$ is normal in $G$ and so $\bar{Q} \leqslant M$, which in turn yields that $[G: M]$ is a prime. Hence we may assume that $Z_{\mathrm{Gn}}(G)$ is a $p$-subgroup. Since $Z_{\mathrm{Gn}}(G)$ is generated by generalized central elements of $G$ and the powers of a generalized central element of $G$ are generalized central elements of $G$, it follows that $G$ contains generalized central elements of order $p$. Let $N$ be the subgroup generated by all such elements. Then $N \triangleleft G$ because a conjugate of a $\pi$ quasinormal subgroup is a $\pi$-quasinormal subgroup. If $N \leqslant M$, then, as before, $[G: M]$ is a prime. On the other hand, if $N \$ M$, then there is an element $y$ of order $p$ such that $\langle y\rangle$ is $\pi$-quasinormal in $G$ and $y \notin M$. Since $P$ is abelian, $\langle y\rangle$ is a normal subgroup of $P$. By (1.4), $p^{\prime}$-elements of $G$ normalize $\langle y\rangle$. Hence $\langle y\rangle \triangleleft G$ and so $M\langle y\rangle$ is a subgroup. This implies that $M\langle y\rangle$ $=G$. Therefore, $[G: M]=|\langle y\rangle|=p$, a prime. Now $G=Z_{\mathrm{Gn}}^{*}(G)$ is super- 
solvable by a theorem of Huppert. This completes the proof.

One can easily verify the next result.

THEOREM 2.8. Let $G$ be a group. Then:

(i) $G$ is supersolvable if and only if $G=Z_{\mathrm{Gn}}^{*}(G)$.

(ii) $G$ is supersolvable if and only if $G / Z_{\mathrm{Gn}}^{*}(G)$ is supersolvable.

THEOREM 2.9. If $S$ is a supersolvable subgroup of a group $G$, then $S Z_{\mathrm{Gn}}^{*}(G)$ is supersolvable.

Proof. We use induction on $|G|$. If $S Z_{\mathrm{Gn}}^{*}(G) \leqslant G$, then $S Z_{\mathrm{Gn}}^{*}\left(S Z_{\mathrm{Gn}}^{*}(G)\right)$ is supersolvable by induction. Proposition 2.4 yields that $Z_{\mathrm{Gn}}^{*}(G)$ $\leqslant Z_{\mathrm{Gn}}^{*}\left(S Z_{\mathrm{Gn}}^{*}(G)\right)$ and so $S Z_{\mathrm{Gn}}^{*}(G)$ is supersolvable. On the other hand, if $S Z_{\mathrm{Gn}}^{*}(G)=G$, then, since

$$
G / Z_{\mathrm{Gn}}^{*}(G)=S Z_{\mathrm{Gn}}^{*}(G) / Z_{\mathrm{Gn}}^{*}(G) \cong S / S \cap Z_{\mathrm{Gn}}^{*}(G)
$$

and $Z_{\mathrm{Gn}}^{*}\left(G / Z_{\mathrm{Gn}}^{*}(G)\right)=1$, it follows that $Z_{\mathrm{Gn}}^{*}\left(S / S \cap Z_{\mathrm{Gn}}^{*}(G)\right)=1$. But $S / S \cap Z_{\mathrm{Gn}}^{*}(G)$ is supersolvable and so

$$
Z_{\mathrm{Gn}}^{*}\left(S / S \cap Z_{\mathrm{Gn}}^{*}(G)\right)=S / S \cap Z_{\mathrm{Gn}}^{*}(G) .
$$

Hence $S / S \cap Z_{\mathrm{Gn}}^{*}(G)=1$. This implies that $S \leqslant Z_{\mathrm{Gn}}^{*}(G)$, which means that $S Z_{\mathrm{Gn}}^{*}(G)=Z_{\mathrm{Gn}}^{*}(G)$. Thus $S Z_{\mathrm{Gn}}^{*}(G)$ is supersolvable.

As a consequence of the last theorem, we have

THEOREM 2.10. $Z_{\mathrm{Gn}}^{*}(G)$ of a group $G$ is contained in the intersection of the maximal supersolvable subgroups of $G$.

Proof. Let $M$ be any maximal supersolvable subgroup of $G$. By Theorem 2.9, $M Z_{\mathrm{Gn}}^{*}(G)$ is supersolvable. Hence either $M Z_{\mathrm{Gn}}^{*}(G)=G$ or $M Z_{\mathrm{Gn}}^{*}(G)$ $=M$. If $M Z_{\mathrm{Gn}}^{*}(G)=M$, then, clearly, $Z_{\mathrm{Gn}}^{*}(G) \leqslant M$. On the other hand, if $M Z_{\mathrm{Gn}}^{*}(G)=G$, then $G$ is supersolvable and so $M=G$. Hence $Z_{\mathrm{Gn}}^{*}(G) \leqslant M$.

REMARK. We have not yet been able to prove or disprove that $Z_{\mathrm{Gn}}^{*}(G)$ is the intersection of the maximal supersolvable subgroups of $G$.

3. Generalized hypercentral subgroups. The notion of hypercentral subgroups, introduced by Baer, is extended. It is shown that the generalized hypercenter is the product of all generalized hypercentral subgroups.

Definition. We shall call a normal subgroup $H$ of a group $G$ a generalized hypercentral ( $G H$-central) subgroup of $G$ if for all $M \leqslant H$ and $M \triangleleft G$, $H / M \cap Z_{\mathrm{Gn}}(G / M) \neq \overline{1}$.

Proposition 3.1. If $H$ is a $G H$-central subgroup of $G$, then for each $N \triangleleft G$ and $N \leqslant H, H / N$ is a $G H$-central subgroup of $G / N$.

Lemma 3.2. If $H \leqslant Z_{\mathrm{Gn}}(G)$ and $H \triangleleft G$, then $H$ is a $G H$-central subgroup of G. In particular, $Z_{\mathrm{Gn}}(G)$ is a $G H$-central subgroup of $G$.

Proof. Let $K \triangleleft G$ and $K \leqslant H$. By $(1.3), Z_{\mathrm{Gn}}(G) K / K=Z_{\mathrm{Gn}}(G) / K$ $\leqslant Z_{\mathrm{Gn}}(G / K)$. Since $H / K \leqslant Z_{\mathrm{Gn}}(G) / K, H / K \cap Z_{\mathrm{Gn}}(G / K)=H / K \neq \overline{1}$.

THEOREM 3.3. For $1 \leqslant i \leqslant m$, every member $\left(Z_{\mathrm{Gn}}(G)\right)_{i}$ of the chain 


$$
\begin{aligned}
1 & =\left(Z_{\mathrm{Gn}}(G)\right)_{0}<Z_{\mathrm{Gn}}(G)=\left(Z_{\mathrm{Gn}}(G)\right)_{1}<\left(Z_{\mathrm{Gn}}(G)\right)_{2} \\
& <\cdots<\left(Z_{\mathrm{Gn}}(G)\right)_{m}=Z_{\mathrm{Gn}}^{*}(G)
\end{aligned}
$$

is a GH-central subgroup of $G$.

Proof. We use induction on $|G|$. In view of the preceding lemma, we prove the theorem for $i=2,3, \ldots, m$. Consider $G / Z_{\mathrm{Gn}}(G)$ and form the chain

$$
\begin{aligned}
\overline{1} & =\left(Z_{\mathrm{Gn}}(G)\right)_{1} / Z_{\mathrm{Gn}}(G)<\left(Z_{\mathrm{Gn}}(G)\right)_{2} / Z_{\mathrm{Gn}}(G) \\
& <\cdots<\left(Z_{\mathrm{Gn}}(G)\right)_{m} / Z_{\mathrm{Gn}}(G)=Z_{\mathrm{Gn}}^{*}(G) / Z_{\mathrm{Gn}}(G),
\end{aligned}
$$

where

$$
\begin{aligned}
& Z_{\mathrm{Gn}}\left(G / Z_{\mathrm{Gn}}(G) /\left(Z_{\mathrm{Gn}}(G)\right)_{i-1} / Z_{\mathrm{Gn}}(G)\right) \\
& \quad=\left(Z_{\mathrm{Gn}}(G)\right)_{i} / Z_{\mathrm{Gn}}(G) /\left(Z_{\mathrm{Gn}}(G)\right)_{i-1} / Z_{\mathrm{Gn}}(G)
\end{aligned}
$$

for $i=2,3, \ldots, m$. Now suppose $M \leqslant\left(Z_{\mathrm{Gn}}(G)\right)_{i}$ and $M \triangleleft G$, for $i>1$. If $Z_{\mathrm{Gn}}(G) \leqslant M$, then

$$
\left(Z_{\mathrm{Gn}}(G)\right)_{i} / Z_{\mathrm{Gn}}(G) / M / Z_{\mathrm{Gn}}(G) \cap Z_{\mathrm{Gn}}\left(G / Z_{\mathrm{Gn}}(G) / M / Z_{\mathrm{Gn}}(G)\right) \neq \overline{1}
$$

since by induction $\left(Z_{\mathrm{Gn}}(G)\right)_{i} / Z_{\mathrm{Gn}}(G)$ is a $G H$-central subgroup of $G / Z_{\mathrm{Gn}}(G)$. This means that $\left(Z_{\mathrm{Gn}}(G)\right)_{i} / M \cap Z_{\mathrm{Gn}}(G / M) \neq \overline{1}$. On the other hand, if $Z_{\mathrm{Gn}}(G) \preccurlyeq M$, then there is a generalized central element $g$ of $G$ such that $g \notin M$. By (1.3), $g M \in Z_{\mathrm{Gn}}(G / M)$ and so $g M \in\left(Z_{\mathrm{Gn}}(G)\right)_{i} / M$ $\cap Z_{\mathrm{Gn}}(G / M)$. Hence $\left(Z_{\mathrm{Gn}}(G)\right)_{i}$ is a $G H$-central subgroup of $G$.

Lemma 3.4. If $N_{1}$ and $N_{2}$ are $G H$-central subgroups of a group $G$, then the product $N_{1} N_{2}$ is also a $G H$-central subgroup of $G$.

Proof. Let $M \triangleleft G$ with $M \leqslant N_{1} N_{2}$. If $M \leqslant N_{1}$, or $M \leqslant N_{2}$, then $N_{1} N_{2} / M \cap Z_{\mathrm{Gn}}(G / M) \neq \overline{1}$ since $N_{i} / M \cap Z_{\mathrm{Gn}}(G / M) \neq \overline{1}$ for $i=1$ or 2 . If $M \cap N_{1}=N_{1}$ and $M \cap N_{2}=N_{2}$, then $N_{1} N_{2} \leqslant M$, which is impossible. Thus we may assume without loss of generality that $M \cap N_{1}=W \leqslant N_{1}$. Since $W$ is also normal in $G, N_{1} / W \cap Z_{\mathrm{Gn}}(G / W) \neq \overline{1}$. Let $x W$ be a nonidentity element of $N_{1} / W \cap Z_{\mathrm{Gn}}(G / W)$. Since $G / M$ is a homomorphic image of $G / W$ and $x \notin M, x M$ is a nonidentity element of $N_{1} N_{2} / M$ $\cap Z_{\mathrm{Gn}}(G / M)$. Hence $N_{1} N_{2}$ is a $G H$-central subgroup of $G$.

From the above results we now obtain the following characterization of the generalized hypercenter.

THEOREM 3.5. $Z_{\mathrm{Gn}}^{*}(G)$ of a group $G$ is the product of all $G H$-central subgroups of $G$.

Proof. Lemma 3.4 yields that the product $P$ of all $G H$-central subgroups of $G$ is $G H$-central. Hence, $Z_{\mathrm{Gn}}^{*}(G) \leqslant P$ by Theorem 3.3. If $Z_{\mathrm{Gn}}^{*}(G) \leqslant P$, then $P / Z_{\mathrm{Gn}}^{*}(G) \cap Z_{\mathrm{Gn}}\left(G / Z_{\mathrm{Gn}}^{*}(G)\right) \neq \overline{1}$ and so $Z_{\mathrm{Gn}}\left(G / Z_{\mathrm{Gn}}^{*}(G)\right) \neq \overline{1}$, an impossibility. Thus $Z_{\mathrm{Gn}}^{*}(G)=P$. 


\section{REFERENCES}

1. R. Baer, The hypercenter of a group. I, Acta Math. 89 (1953), 165-208. MR 15, 395.

2. — Das Hyperzentrum einer Gruppe. II, Arch. Math. 4 (1953), 86-96. MR 15, 396.

3. - Das Hyperzentrum einer Gruppe. III, Math. Z. 59 (1953), 299-338. MR 15, 598.

4. - Group elements of prime power index, Trans. Amer. Math. Soc. 75 (1953), 20-47. MR $14,1059$.

5. W. E. Deskins, On quasinormal subgroups of finite groups, Math. Z. 82 (1963), 125-132. MR 27 \#3699.

6. B. Huppert, Endliche Gruppen. I, Die Grundlehren der math. Wissenschaften, Band 134, Springer-Verlag, Berlin and New York, 1967. MR 37 \#302.

7. O. H. Kegel, Sylow-Gruppen und Subnormalteiler endlicher Gruppen, Math. Z. 78 (1962), 205-221. MR 26 \#5042.

8. N. P. Mukherjee, The hyperquasicenter of a finite group, Ph.D. Thesis, Michigan State University, East Lansing, Mich., 1968.

9. - The hyperquasicenter of a finite group. I, Proc. Amer. Math. Soc. 26 (1970), 239-243. MR 42 \#3166.

10. O. Ore, Contributions to the theory of groups of finite order, Duke Math. J. 5 (1939), 431-460.

Department of Mathematics, Ferris State College, Big Rapids, Michigan 49307 